\title{
Resection of ascending aortic aneurysm without use of an interposition aortic graft
}

\author{
John S. Ikonomidis, MD, PhD, FRCS(C), ${ }^{*}$ Abe DeAnda, Jr, MD, ${ }^{* *}$ and D. Craig Miller, MD, Stanford, Calif
}

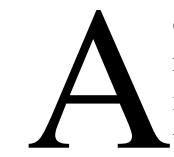

ortic resection or reinforcement followed by primary repair or anastomosis of the aorta has relatively few indications. These include coarctation, interrupted aortic arch, traumatic aortic rupture, ${ }^{1}$ and, in the past, acute aortic dissection. ${ }^{2}$ In general, aortic aneurysms are repaired

\footnotetext{
From the Department of Cardiovascular and Thoracic Surgery, Stanford University Medical School, Stanford, Calif.

Received for publication Dec 5, 2000; accepted for publication Dec 21, 2000.

Address for reprints: D. Craig Miller, MD, Falk Cardiovascular Research Center, Stanford University Medical School, Stanford, CA 94305-5247 (Email: dcm@stanford.edu).

${ }^{*}$ Current address: Division of Cardiothoracic Surgery, Medical University of South Carolina, Charleston, SC.

${ }^{* *}$ Current address: Division of Cardiothoracic Surgery, Medical College of Virginia, Richmond, Va.

J Thorac Cardiovasc Surg 2001;122:395-7

Copyright $\odot 2001$ by The American Association for Thoracic Surgery $0022-5223 / 2001 \$ 35.00+0 \quad \mathbf{1 2 / 5 4 / 1 1 3 9 2 7}$

doi:10.1067/mtc.2001.113927
}

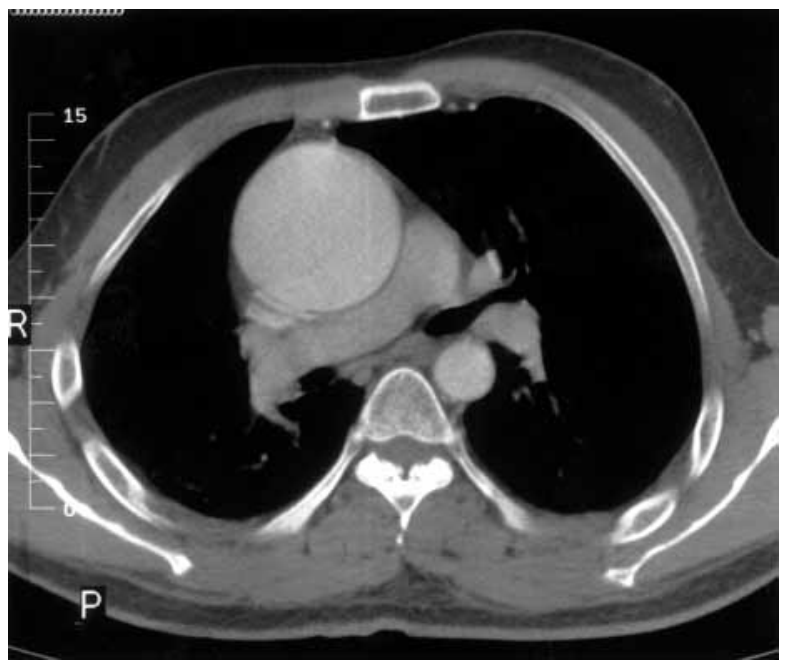

Figure 1. CT scan showing a large ascending aortic aneurysm at the level of the right main pulmonary artery. Note the marked compression of the superior vena cava. 


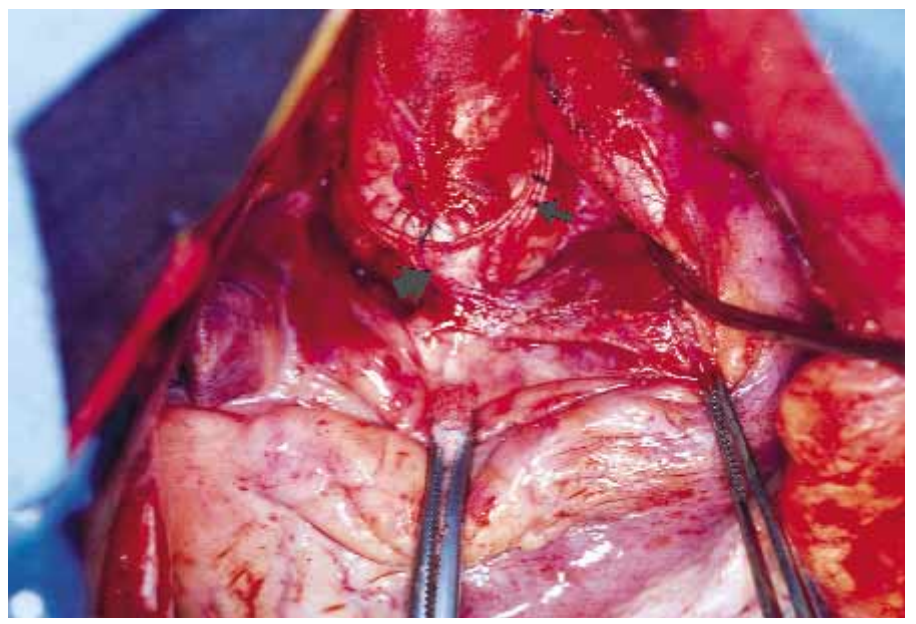

Figure 2. Intraoperative photograph showing the completed primary end-to-end aortic anastomosis. Small arrow shows the ring of Dacron graft used to remodel the sinotubular junction. Large arrow shows the large right coronary artery, which arose somewhat higher than normal.

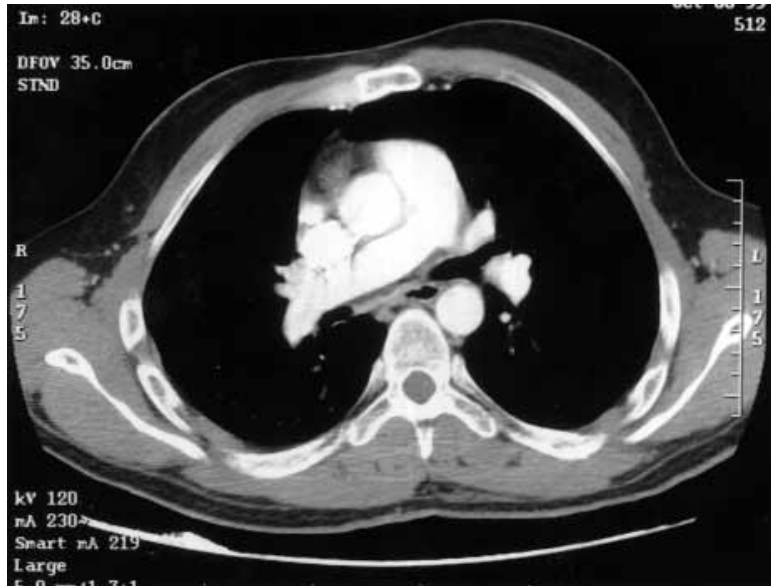

Figure 3. Postoperative CT scan showing the ascending aortic aneurysm repair at the same level as Figure 1 . The superior vena cava no longer is extrinsically compressed.

by placement of an aortic interposition graft. We describe the case of a patient with a large ascending aortic aneurysm who was treated by resection of the aneurysm, remodeling of the sinotubular junction, and primary end-to-end aortic anastomosis without the need for graft replacement. Follow-up out to 21 months showed no subsequent aortic dilatation or aortic valvular regurgitation.

\section{Clinical Summary}

A previously healthy 34-year-old man with a history of mild hypertension had a flulike illness with a cough and underwent a chest radiograph, which showed a markedly widened mediastinum. A computed tomographic (CT) scan of the chest revealed a $7.0-\mathrm{cm}$ ascending aortic aneurysm without involvement of the sinuses of Valsalva or arch. Two weeks later, he returned with sudden, severe chest pain and underwent a second CT scan (Figure 1),

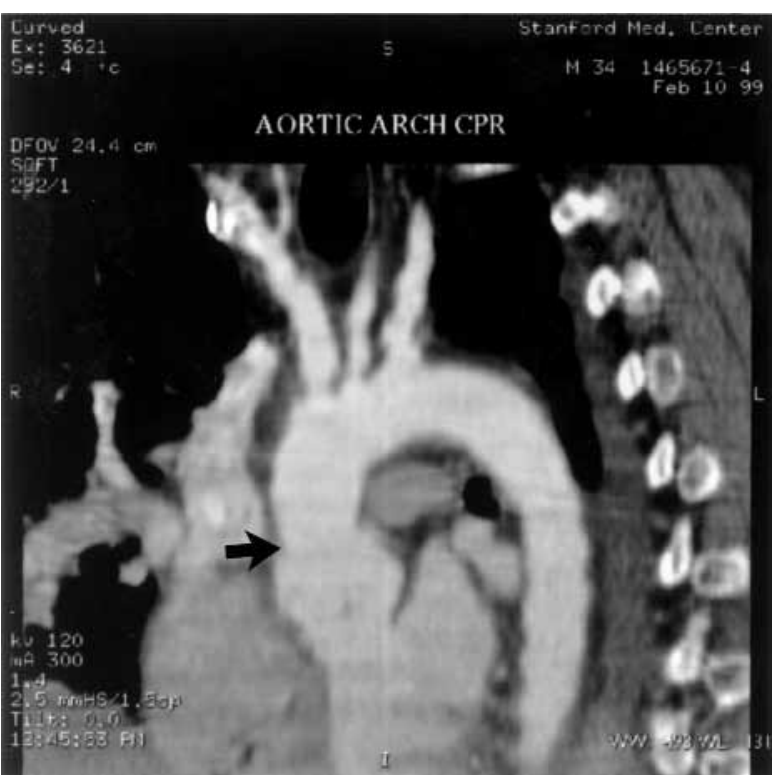

Figure 4. Curved planar reformat 3-dimensional reconstruction of postoperative CT scan in the right posterior oblique projection showing the reconstructed ascending aorta and sinuses of Valsalva. Arrow indicates the remodeled sinotubular junction.

which showed no evidence of aortic dissection or rupture, no change in the size of the aneurysm, and no evidence of other pathologic conditions within the chest. The chest pain was believed to be related to the aneurysm, and the patient was transferred to Stanford Medical Center by AirEvac on an emergency basis.

On physical examination, the patient was pain free and in hemodynamically stable condition. He was tall and had some Marfanoid features, including long arms. No family history was available because he was adopted. He had previously undergone 
bilateral inguinal hernia repair as an adolescent. The only other notable physical findings were a tall, narrow hard palate and a grade 1/6 murmur of aortic regurgitation. There was no lens dislocation. Laboratory and electrocardiographic assessment were within normal limits.

At operation, there was no evidence of leak or of acute or chronic inflammation. The large aneurysm involved most of the ascending aorta but narrowed to normal caliber $4 \mathrm{~cm}$ proximal to the origin of the innominate artery; therefore, simple distal arch cannulation with crossclamping of the aorta obliquely under the innominate artery was used instead of profound hypothermic circulatory arrest. Intraoperative transesophageal echocardiography showed a normal-caliber aortic arch and descending thoracic aorta. There was moderate dilatation of the aortic sinuses with mild central aortic regurgitation. The mitral valve was normal. The aneurysm was resected from the sinotubular junction to the grossly normal size distal ascending aorta. The aortic wall appeared normal (1.5-2 mm thick), and there was no sign in the intima of aortitis, fibrous plaque, or atherosclerosis. The sinotubular junction diameter was dilated $(35 \mathrm{~mm})$ because of the ascending aneurysm. The aortic valve was tricuspid, and the aortic anulus measured 25 $\mathrm{mm}$. The leaflets were normal, the average leaflet height being 17 $\mathrm{mm}$. Using the formula of Feindel and David, ${ }^{3} \mathrm{~d}=\left(\left[\mathrm{h}_{\text {leaflet }} \cdot 2\right]\right.$ $0.67)+\left(2 \mathrm{~h}_{\text {Ao wall }}\right)$, a 1-cm ring of a $26-\mathrm{mm}$ woven double-velour Hemashield Dacron graft (Meadox Medicals, Medi-Tech; Boston Scientific Corp, Natick, Mass) with scallops excised to fit around the left and right coronary arteries was slipped around the proximal aortic root and sutured circumferentially to the sinotubular ridge with 4-0 polypropylene to reduce its diameter. At this point it became apparent that the remodeled sinotubular ridge and the distal ascending aorta were nearly opposed with little tension. Given this finding and the normal integrity of the aorta, we decided that the use of a graft was unnecessary. The primary aortic anastomosis was then performed with 4-0 polypropylene (Figure 2). No aortic regurgitation was apparent on transesophageal echocardiography after weaning from cardiopulmonary bypass. A baseline postoperative CT angiogram showed a normal contour of the sinuses of Valsalva and ascending aortic (Figures 3 and 4); transthoracic echocardiography showed no evidence of aortic regurgitation. Pathologic examination of the aortic tissue revealed only fibroatheromatous changes in the aneurysm wall without major disruption of the elastic lamina; colloidal iron staining did not demonstrate any pools of mucopolysaccharides. The patient recovered uneventfully and was discharged on postoperative day 4 on a regimen of aspirin and metropolol. He has done well for 21 months except for some activity-related, nonspecific sternal and shoulder discomfort and the ocular migraine syndrome, accompanied by transient visual disturbances (without documented retinal emboli), and rare presyncopal spells, for which he currently is undergoing an epilepsy work-up. Formal genetics evaluation concluded that he probably had some uncharacterized connective tissue disorder but did not satisfy the formal Ghent criteria for the
Marfan syndrome. Follow-up CT scans at 8 and 16 months after repair show no evidence of recurrent aneurysmal disease, and a follow-up repeat echocardiogram at 16 months again demonstrated no aortic regurgitation.

\section{Discussion}

Primary anastomosis or repair of the aorta is considered the standard treatment for certain congenital anomalies such as coarctation and interrupted aortic arch. Primary repair has also been used occasionally in traumatic aortic injuries, ${ }^{1}$ and a lateral aneurysmorrhaphy repair has been used rarely in special cases of suprarenal abdominal aortic aneurysms. ${ }^{4}$ In the past, acute aortic dissection has been treated by means of a similar primary repair technique, ${ }^{2}$ but current evidence indicates that this strategy does not provide durable results. ${ }^{5}$

Aortic interposition grafting is virtually always required after resection of ascending and descending thoracic aortic aneurysms given the key technical points of relieving tension at the anastomosis and reinforcement of the inherently weak aortic wall. If approximation of the aorta after aneurysm excision can be accomplished without undue tension (especially in young patients with more mobile and elastic aortas) and there is no question concerning the structural integrity of the aortic wall, this anecdotal case suggests that a primary anastomosis might be prudent in highly selected patients. Recently, 10 cases of primary end-to-end aortic anastomosis for ascending aneurysm were reported in abstract form ${ }^{6}$ and involved concomitant procedures in 8. Within the average follow-up interval of 11 months (maximal follow-up 25 months), no treatment failures occurred. However, no data exist regarding the long-term durability of this approach. Until more definite late information is known, this technique should be reserved for special circumstances and probably not applied too widely.

\section{References}

1. Stemmed CA, Wood MN, Razor AJ, Killeen JD, Gan KA. Primary repair of traumatic aortic rupture: a preferred approach. $J$ Trauma. 1992;32:588-92.

2. Olinger GN, Schweiger JA, Galbraith TA. Primary repair of acute ascending aortic dissection. Ann Thorac Surg. 1987;44:389-93.

3. David TE, Feindel CM. An aortic valve-sparing operation for patients with aortic incompetence and aneurysm of the ascending aorta. $J$ Thorac Cardiovasc Surg. 1992;103:617-22.

4. Lang SJ, Gewirtz HS, Baker JD. Suprarenal abdominal aortic aneurysm managed by excision and primary repair using a lateral suture technique. J Cardiovasc Surg (Torino). 1985;26:595-7.

5. Pugilese P, Pessotto R, Santini F, Montalbano G, Luciani GB, Mazzucco A. Risk of late operations in patients with acute type A aortic dissection: impact of a more radical surgical approach. Eur $J$ Cardiothoracic Surg. 1998;13:576-80.

6. Zehr, KJ, Sternik L, Schaff HV. Resection and primary end-to-end anastomosis of ascending aortic aneurysms. Circulation. 2000;102(Suppl):II-400-1. 